\title{
Identification of a blood-borne miRNA signature of synovial sarcoma
}

Alba Fricke1, Prisca V. Ullrich¹, Jürgen Heinz², Dietmar Pfeifer ${ }^{2}$, Jutta Scholber ${ }^{3}$, Georg W. Herget ${ }^{4}$, Oliver Hauschild ${ }^{4}$, Peter Bronsert ${ }^{5,6}$, G. Björn Stark' , Holger Bannasch¹, Steffen U. Eisenhardt ${ }^{{ }^{*}+}$ and David Braig ${ }^{1 \dagger}$

\begin{abstract}
Background: Synovial sarcoma account for approximately $10 \%$ of all soft-tissue tumors and occur most frequently in young adults. A specific translocation in this sarcoma induces fusion of the SYT gene on chromosome 18 to the SSX genes on chromosome $X$, leading to proliferation of the tumor cells. The need for non-invasive biomarkers indicating recurrence and activity of this disease has sparked research into short non-coding RNA known as microRNA (miRNA).

Methods: Blood samples of patients with active synovial sarcoma and of synovial sarcoma patients in complete remission as well as of healthy donors and patients with active leiomyosarcoma, MPNST, Ewing sarcoma and liposarcoma were collected. Whole blood RNA was extracted and samples of patients with active synovial sarcoma and of healthy donors were analyzed using an Affymetrix GeneChip miRNA Array v. 4.0. qRT-PCR was carried out to confirm a panel of miRNAs which where differentially expressed in the miRNA array. This miRNA-panel was further evaluated in patients with synovial sarcoma in complete remission and patients with active leiomyosarcoma, MPNST, Ewing sarcoma and liposarcoma as well as in an independent cohort of synovial sarcoma patients.

Results: Unsupervised hierarchical clustering of the miRNA arrays separated patients with active synovial sarcoma from healthy controls. A panel of seven miRNAs (miR-99a-5p, miR-146b-5p, miR-148b-3p, miR-195-5p, miR-223-3p, miR-500b-3p and miR-505-3p) was further validated by qRT-PCR to be significantly upregulated in synovial sarcoma patients. Moreover, most of the analyzed miRNAs were shown to be significantly upregulated in synovial sarcoma patients compared to leiomyosarcoma, MPNST, Ewing sarcoma and liposarcoma patients. Validation of the miRNA panel in an independent cohort of synovial sarcoma patients confirmed higher expression levels compared to healthy controls and patients in complete remission.

Conclusion: Our results have identified a specific whole blood miRNA signature that may serve as an independent biomarker for the diagnosis of local recurrence or distant metastasis of synovial sarcoma. It even distinguishes synovial sarcoma from other sarcoma subtypes, thus potentially serving as a specific biomarker for synovial sarcoma.
\end{abstract}

Keywords: Whole-blood-RNA, miRNA, Synovial sarcoma, Biomarker, Liquid biopsy

\footnotetext{
*Correspondence: steffen.eisenhardt@uniklinik-freiburg.de

${ }^{\dagger}$ Equal contributors

1 Department of Plastic and Hand Surgery, University Medical Center Freiburg,

Hugstetter Strasse 55, 79106 Freiburg, Germany

Full list of author information is available at the end of the article
} 


\section{Background}

Soft tissue sarcoma constitute a heterogeneous group of malignant tumors of mesenchymal origin, showing frequent local recurrence and distant metastasis [1, 2]. Synovial sarcoma account for approximately $10 \%$ of all soft-tissue sarcoma and most frequently develop in the extremity of young adults [3]. The cytogenetically defined translocation $t(X ; 18)$ (p11.2; q11.2) found in synovial sarcoma results in the fusion of the SYT gene on chromosome 18 to either SSX1, SSX2 or SSX4 on chromosome $\mathrm{X}$, resulting in the formation of a SS18SSX1, SS18-SSX2 or the rare SS18-SSX4 fusion transcript [4-7], which allow a very specific and sensitive molecular diagnosis of synovial sarcoma by fluorescence in situ hybridization (FISH) or quantitative and conventional reverse transcription-polymerase chain reaction (RT-PCR) of tumor tissue [8]. However, diagnosis of local recurrence or distant metastasis of synovial sarcoma is restricted to magnetic resonance imaging (MRI), computed tomography (CT) and biopsy [2]. Therefore, the need for non-invasive biomarkers indicating recurrence and activity of this disease has sparked research into microRNAs (miRNAs), which are small, non-coding molecules of about 22 nucleotides in length. MiRNAs regulate the expression of target genes through mRNA degradation and translation inhibition [9], and have been shown to serve as potential biomarkers in different malignancies [10-12].

Interestingly, many poorly differentiated tumors can be successfully classified using miRNA expression profiles whereas messenger RNA profiles may be rather inaccurate when applied to the same samples [13]. Recent publications show that sarcoma tissue of different histological subtypes show distinct miRNA expression profiles [14-16]. However, few studies concentrate on peripheral blood miRNA profiling. Miyachi et al. detected miR-206 to be significantly upregulated in peripheral blood of patients with rhabdomyosarcoma compared to patients with non-rhabdomyosarcoma tumors [17], thus showing that miRNA expression levels may be used to differentiate between different tumors. Furthermore, the detection of miRNAs in peripheral blood may serve as a non-invasive method for diagnosis of metastasis or local tumor recurrence, possibly improving survival rates due to earlier detection of sarcoma recurrence as well as making follow-up more economical. Therefore, we performed a comprehensive microarray-based miRNA screen of whole blood RNA of synovial sarcoma patients compared to healthy controls, followed by validation of deregulated miRNAs using quantitative Real-Time polymerase chain reaction (qRTPCR), assessing differences in patients with active synovial sarcoma compared to healthy controls, patients with synovial sarcoma in complete remission and patients with active leiomyosarcoma, malignant peripheral nerve sheath tumor (MPNST), Ewing sarcoma and liposarcoma.

\section{Results and discussion}

The screening cohort of patients with active synovial sarcoma $(\mathrm{n}=5)$ as analyzed in a microarray as well as the independent validation cohort of synovial sarcoma patients $(n=3)$ did not differ significantly from the healthy controls $(n=5)$, synovial sarcoma in complete remission $(\mathrm{n}=10)$ and active leiomyosarcoma $(\mathrm{n}=5)$, MPNST $(n=3)$, Ewing sarcoma $(n=3)$ and liposarcoma $(n=6)$ group concerning age, BMI, hemoglobin $(\mathrm{Hb})$ level, platelet count and leukocyte count (Additional file 1 and Additional file 2). Two patients with active synovial sarcoma presented the SS18-SSX2 fusion gene phenotype, while five presented the more common SS18-SSX1 phenotype $[6,18]$. Tumor tissue of one patient was not available for analysis. Information regarding disease and therapy status of sarcoma patients is depicted in Additional file 3. Whole blood RNA from patients with active synovial sarcoma $(n=5)$ and healthy controls $(n=5)$ was analyzed by Affymetrix miRNA 4.0 Arrays. Microarray data are available in the ArrayExpress database (www.ebi.ac.uk/arrayexpress) under accession number E-MTAB-3273 (http://www.ebi.ac.uk/arrayexpress/experiments/E-MTAB-3273). Hierarchical clustering of all covered human mature miRNAs and human premiRNAs separated sarcoma samples from control samples (Fig. 1a). Following hierarchical clustering, the array results were narrowed down to deregulated miRNAs with a fold change $>|3.0|$, a $p$-value $<0.005$, a qvalue (false discovery rate (FDR)-corrected $p$-value) [19] $<0.2$ and a similar deregulation of related miRNAs with the same base sequence throughout the array. Of the ten miRNAs, which met these criteria (miR-99a-5p, miR-146b-5p, miR-148b-3p, miR-183-3p, miR-195-5p, miR-223-3p, miR-500b-3p, miR-505-3p, miR-589-5p and miR-1225-5p), nine miRNAs showed an increased expression in tumor patients and one miRNA was decreased (Table 1). Unsupervised hierarchical clustering of this panel could again separate sarcoma patients from healthy controls (Fig. 1b).

Subsequently, seven miRNAs were further validated by qRT-PCR (Fig. 2): miR-99a-5p, miR-146b-5p, miR-148b3p, miR-195-5p, miR-223-3p, miR-500b-3p and miR505-3p. All seven miRNAs were significantly upregulated in the screening cohort of patients with synovial sarcoma $(n=5)$ compared to healthy controls $(n=5)$. Moreover, five of the analyzed miRNAs were shown to be significantly upregulated in the screening cohort of active synovial sarcoma patients compared to patients with active leiomyosarcoma $(n=5)$ : miR-146b-5p, miR148b-3p, miR-223-3p, miR-500b-3p and miR-505-3p. 


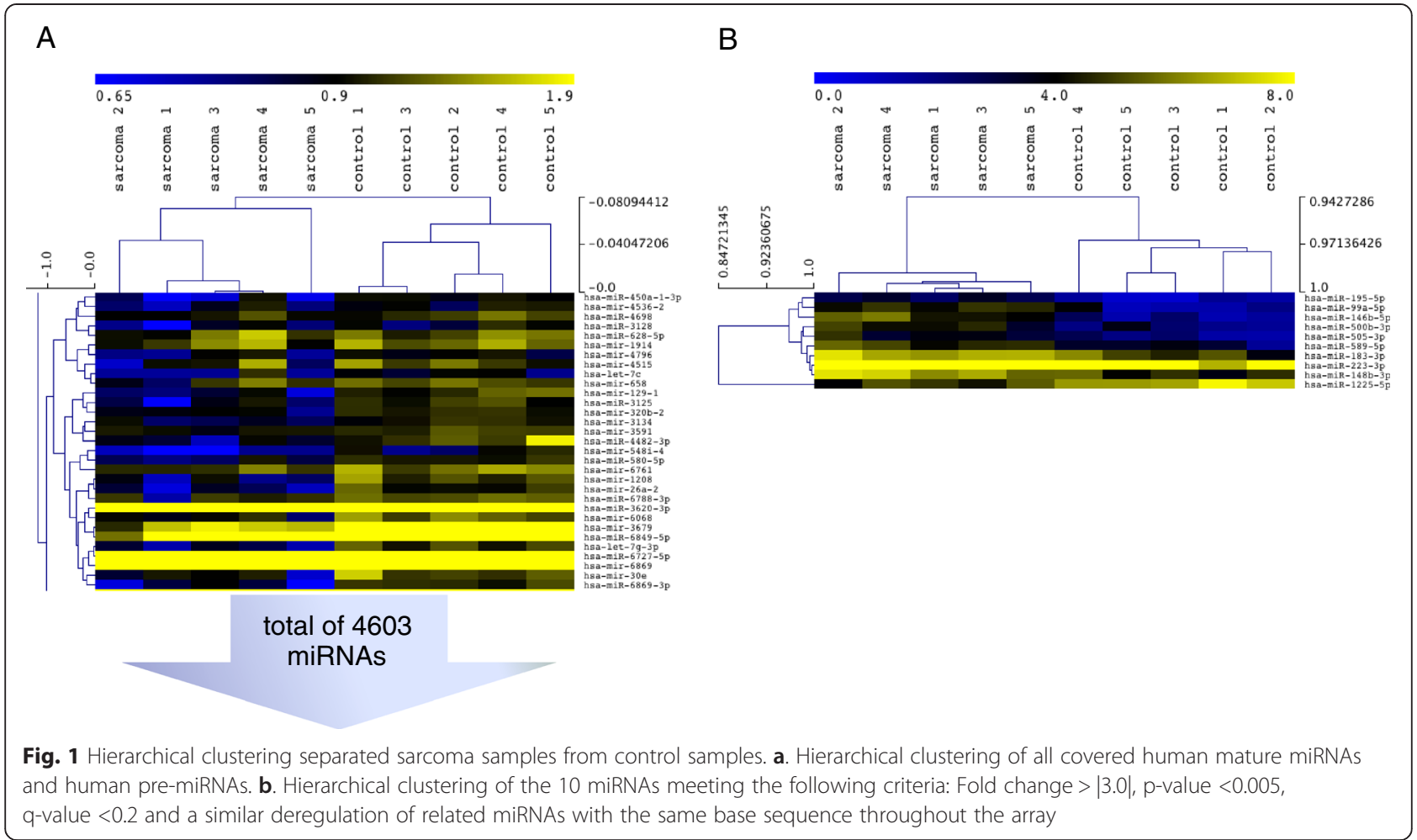

MiR-99a-5p and miR-195-5p were not significantly deregulated (Fig. 2). Comparing the screening cohort of active synovial sarcoma patients to patients with active MPNST ( $\mathrm{n}=3$ ), six of the analyzed miRNAs were shown to be significantly upregulated: miR-99a-5p, miR-146b5p, miR-148b-3p, miR-223-3p, miR-500b-3p and miR505-3p. MiR-195-5p was not significantly deregulated (Fig. 2). Analyzing active synovial sarcoma patients and patients with active Ewing sarcoma $(n=3)$, five of the

Table 1 MiRNAs with a fold change of $>|3.0|$, a $p$-value $<0.005$, a q-value $<0.2$ and a similar deregulation of related miRNAs with the same base sequence throughout the array

\begin{tabular}{llllll}
\hline Transcript ID & $\begin{array}{l}\text { Sarcoma } \\
\text { Mean }\end{array}$ & $\begin{array}{l}\text { Sarcoma } \\
\text { STD }\end{array}$ & $\begin{array}{l}\text { Control } \\
\text { Mean }\end{array}$ & $\begin{array}{l}\text { Control } \\
\text { STD }\end{array}$ & $\begin{array}{l}\text { Fold } \\
\text { Change }\end{array}$ \\
\hline upregulated & & & & & \\
hsa-miR-146b-5p & 4.83 & 0.85 & 1.81 & 0.65 & 8.10 \\
hsa-miR-99a-5p & 4.51 & 0.35 & 1.92 & 1.17 & 6.03 \\
hsa-miR-223-3p & 10.50 & 0.66 & 8.25 & 0.91 & 4.77 \\
hsa-miR-148b-3p & 6.68 & 0.82 & 4.58 & 0.74 & 4.29 \\
hsa-miR-500b-3p & 4.28 & 0.65 & 2.28 & 0.82 & 4.01 \\
hsa-miR-183-3p & 6.83 & 0.46 & 4.97 & 0.90 & 3.62 \\
hsa-miR-589-5p & 4.84 & 0.57 & 3.06 & 0.82 & 3.43 \\
hsa-miR-505-3p & 3.73 & 0.67 & 1.96 & 0.61 & 3.40 \\
hsa-miR-195-5p & 2.96 & 0.43 & 1.24 & 0.46 & 3.30 \\
downregulated & & & & & -4.39 \\
hsa-miR-1225-5p & 4.76 & 0.64 & 6.89 & 0.64 & \\
\hline
\end{tabular}

analyzed miRNAs were shown to be significantly upregulated: miR-146b-5p, miR-148b-3p, miR-223-3p, miR500b-3p and miR-505-3p. MiR-99a-5p and miR-195-5p were not significantly deregulated. Compared to patients with active liposarcoma $(n=6)$, all seven analyzed miRNAs were shown to be significantly upregulated (Fig. 2). These results suggest that the detection of these miRNAs in whole blood of synovial sarcoma patients provides a non-invasive way to detect distant metastasis or local recurrence and this "liquid biopsy" could potentially develop into a further keystone of tumor diagnosis and staging. We therefore validated the expression levels of all seven miRNAs by qRT-PCR in an independent cohort of active synovial sarcoma patients $(n=3)$ : Five of the analyzed miRNAs were shown to be significantly upregulated when compared to the group of healthy donors (miR-146b-5p, miR-148b-3p, miR-195-5p, miR500b-3p and miR-505-3p). MiR-99a-5p and miR-223-3p were also expressed in higher quantities, however differences were not statistically significant (Fig. 3). Expression of this miRNA panel was further analyzed in samples of synovial sarcoma patients in complete remission $(n=10)$. All seven miRNAs were significantly upregulated in the independent cohort of active synovial sarcoma patients compared to patients with synovial sarcoma in complete remission (Fig. 3)

To exclude a bias of fundamental expression levels of miRNAs among the individual patients with synovial sarcoma and to illustrate the change of miRNA expression at 


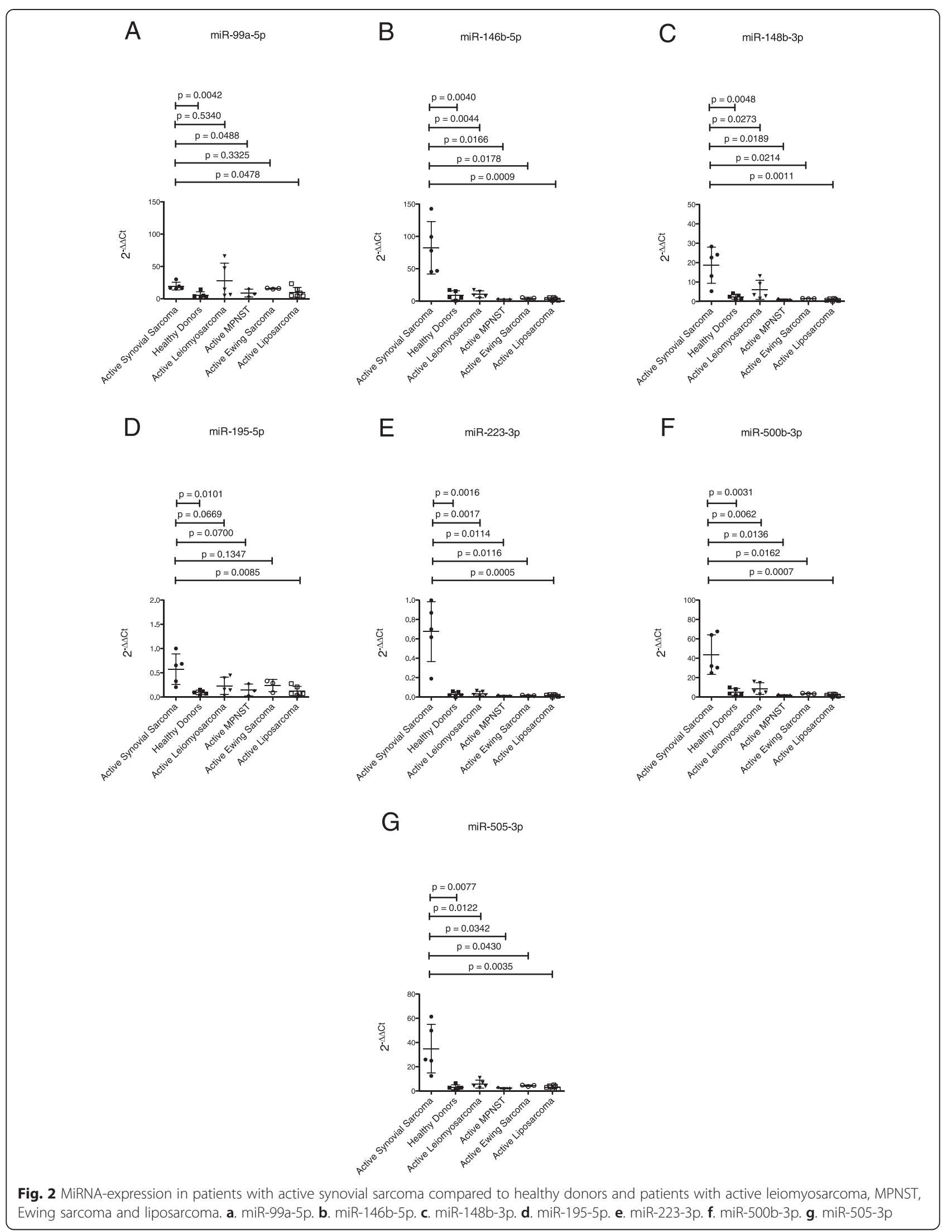




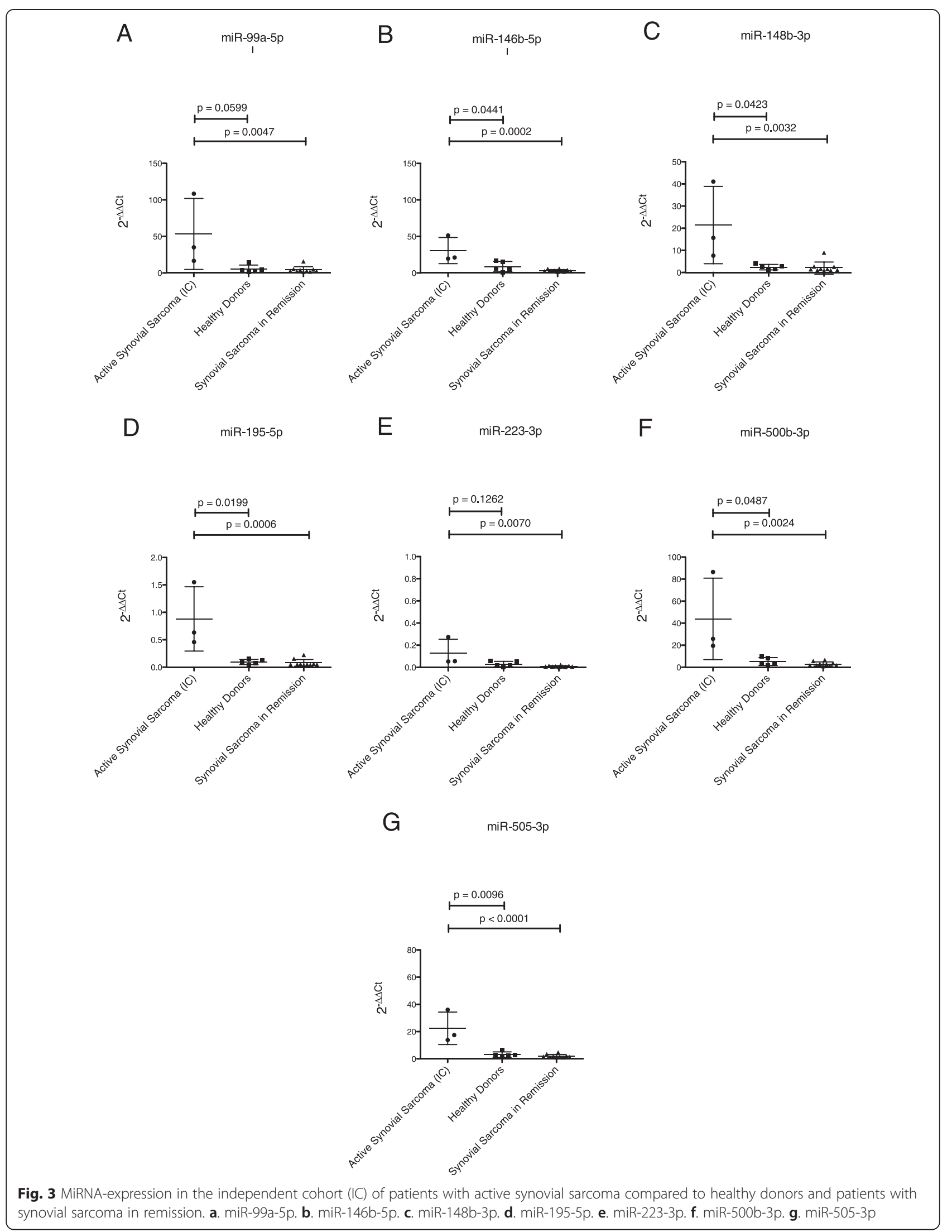


remission and in active disease, we performed a matchedpair analysis in two individual sarcoma patients. Patient 1 initially presented with localized disease of the lower extremity. The miRNA-expression before surgical resection of the tumor as well as two weeks, three months and ten months after complete surgical removal of the tumor was analyzed. The miRNA expression levels decreased rapidly after tumor resection (Fig. 4). Patient 2 was part of the synovial sarcoma in remission cohort at first with unsuspicious miRNA levels. However, she developed pulmonary metastases four months after the initial blood withdrawal. The 2nd blood withdrawal was carried out 5 month after the 1 st blood withdrawal. At this time, the miRNA expression levels were significantly elevated when compared to their expression at the time of remission (Fig. 4). Thus, by performing a matched-pair analysis in these two individual sarcoma patients, we were able to illustrate the downregulation of miRNA expression at remission as well as the upregulation of the presented miRNAs in reoccurring active disease.

In summary, the detected miRNAs might provide sensitive biomarkers to distinguish patients with active synovial sarcoma from healthy controls and patients in complete remission by a simple and non-invasive blood test. They might also be useful to distinguish synovial sarcoma from other soft tissue sarcoma subtypes.

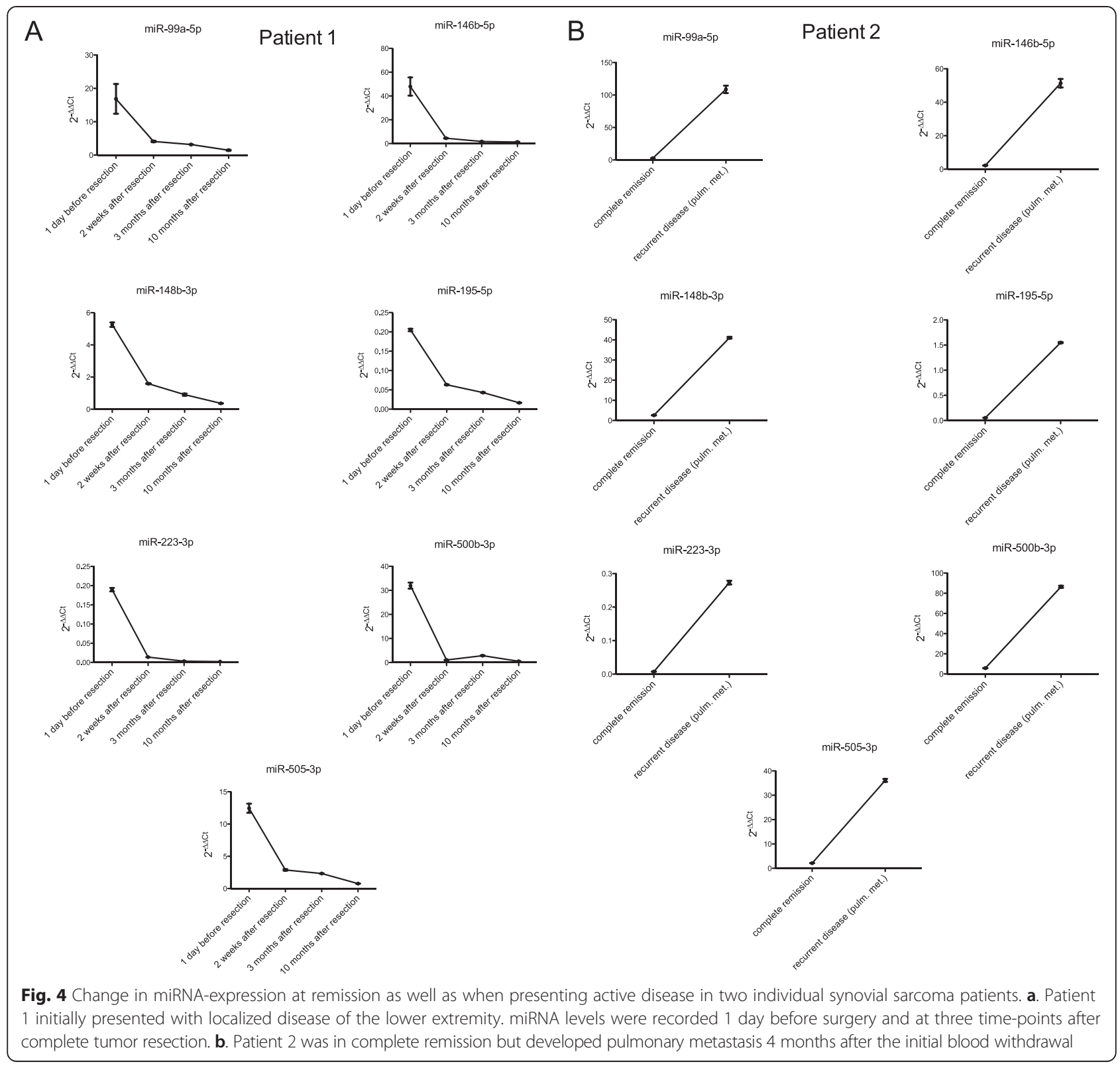


Expression profiling of miRNAs in whole blood may be altered by differences in circulating blood cells. In our two cohorts, patients with active synovial sarcoma did not differ significantly from the healthy controls, synovial sarcoma in complete remission and active leiomyosarcoma, MPNST, Ewing Sarcoma and liposarcoma group concerning age, BMI, hemoglobin-level, platelet count and leukocyte count (Additional file 1 and Additional file 2), thus showing that different miRNA expression in the patient groups evaluated was not due to changes in cell populations such as cancer- and chemotherapy-induced anemia. Furthermore, miRNAexpression levels did not differ whether patients received anticoagulation or chemotherapy. The fact that the patient receiving radiotherapy showed the lowest miRNAexpression levels of all active synovial sarcoma patients for most of the detected miRNAs (Additional file 4) was probably due to the fact that this patient was also the only patient presenting with localized disease, thus suggesting that high miRNA expression levels reflect the dissemination or possibly the activity of the disease. When analyzing miRNA expression levels in synovial sarcoma tissue and whole blood of patients with active synovial sarcoma, the majority of the detected miRNAs did not show correlations with SS18-SSX1 and 2 fusion gene phenotypes (Additional file 5). However, the sample number in these subgroups are to low to allow for a valid conclusion. A major advantage of blood sample collection in PAXgene Blood RNA Tubes is the immediate stabilization of intracellular RNA, thus preserving the gene expression profile for reliable downstream gene expression analysis. Without immediate stabilization, degradation of RNA and upregulation or downregulation of transcripts may alter the results of further analysis. Furthermore, blood samples collected in PAXgene Blood RNA Tubes can be safely stored or transported at room temperature for up to $72 \mathrm{~h}$ and at $-20{ }^{\circ} \mathrm{C}$ or $-70{ }^{\circ} \mathrm{C}$ for at least 50 months. This might reduce pre-analytical errors and facilitate future multi-centre studies, as immediate processing, which is for example needed for the detection of plasma biomarkers, is not necessary, thus avoiding potential changes in miRNA levels by different processing conditions [20].

Keller et al., analyzing 454 whole blood samples collected in PAXgene Blood RNA Tubes from human individuals with different cancers or non-cancer diseases, were able to predict the correct disease in $67.45 \%$ of all individuals by their miRNA expression profiles. Furthermore, they were able to distinguish lung cancer from chronic obstructive pulmonary disease (COPD) with an accuracy of $91.7 \%$, thus providing support for the feasibility of peripheral whole blood miRNA expression patterns in spite of the fact that changes in cell populations may affect overall miRNA profiles. They also found that only a few of the miRNAs deregulated in blood were also previously reported as deregulated in solid tissues derived from individuals with the same diseases [21], which explains why the miRNAs found to be deregulated in whole blood of synovial sarcoma patients in our study did not correlate with previous findings of deregulated miRNAs such as miR-183, miR-200b*, miR-375, [16] let-7e, miR-99b, and miR-125-3p, [18] miR-200b, miR-200c and miR-141 [15] in synovial sarcoma tissue. This might be due to the fact that the miRNA expression patterns found in whole blood depict the miRNA expression patterns of peripheral blood mononuclear cells (PBMC) rather than the miRNA expression patterns of circulating tumor cells, as Mookherjee et al. proved in their study [22]. Furthermore, Leidinger et al. showed that especially the miRNA signature of cells of the innate immune system allowed separation between lung cancer patients and healthy controls, however pointing out that cancer-specific miRNA expression of whole blood samples are not determined by a single cell type but influenced by other blood constituents such as platelets or erythrocytes [23]. These studies point out that the miRNA signature of synovial sarcoma detected in our study represents a response of the innate immune system to the tumor rather than the miRNA expression profile of the tumor itself, thus explaining why none of the presented miRNAs match the highly expressed miRNAs in synovial sarcoma tissues as described by Fujiwara et al. [24], Hisaoka et al. [18] and Renner et al. [16]. This is further suggested by the following findings: When comparing the expression of the detected miRNAs in whole blood RNA of patients with active synovial sarcoma with the corresponding tumor tissue $(n=7)$, we found that most miRNAs were significantly higher expressed in whole blood RNA of patients with active synovial sarcoma (Fig. 5). MiR-99a-5p was the only miRNA being higher expressed in synovial sarcoma tissue than in whole blood. Comparing the miRNA expression levels in synovial sarcoma tissue $(n=7)$ with healthy skeletal muscle $(n=5)$, we found no significantly different expression of most of the detected miRNAs (Fig. 5). Only MiR-223-3p was significantly higher expressed in healthy skeletal muscle tissue (Fig. 5e). When comparing the miRNA expression levels of lymphocytes (Jurkat; human $\mathrm{T}$ lymphocyte cell line) and monocytes (THP-1; human acute monocytic leukemia cell line) to synovial sarcoma cells (SYO-1 und 1273/99 cell line), we found that most miRNAs were significantly higher expressed in human lymphocytes compared to SYO-1 and 1273/99 synovial sarcoma cells and human monocytes compared to SYO-1 and 1273/99 synovial sarcoma cells (Additional file 6). Interestingly, miR-99a$5 p$ is the only miRNA being higher expressed both in synovial sarcoma tissue compared to whole blood cells 

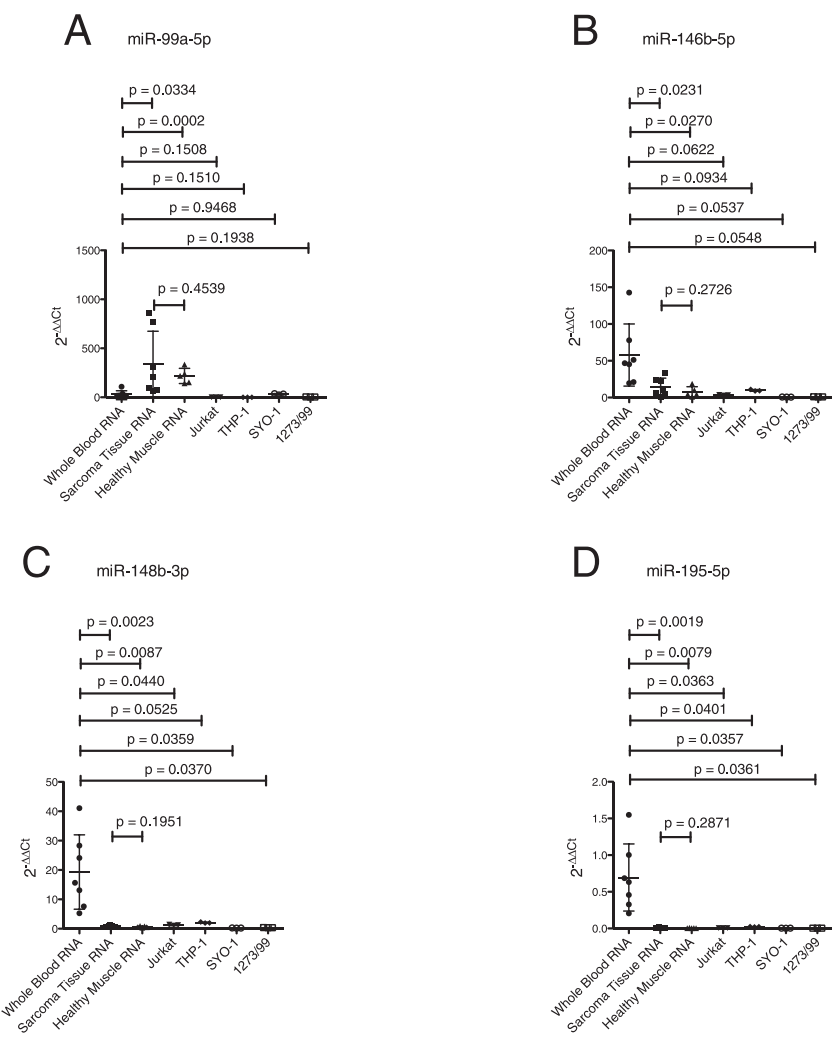

D miR-195-5p
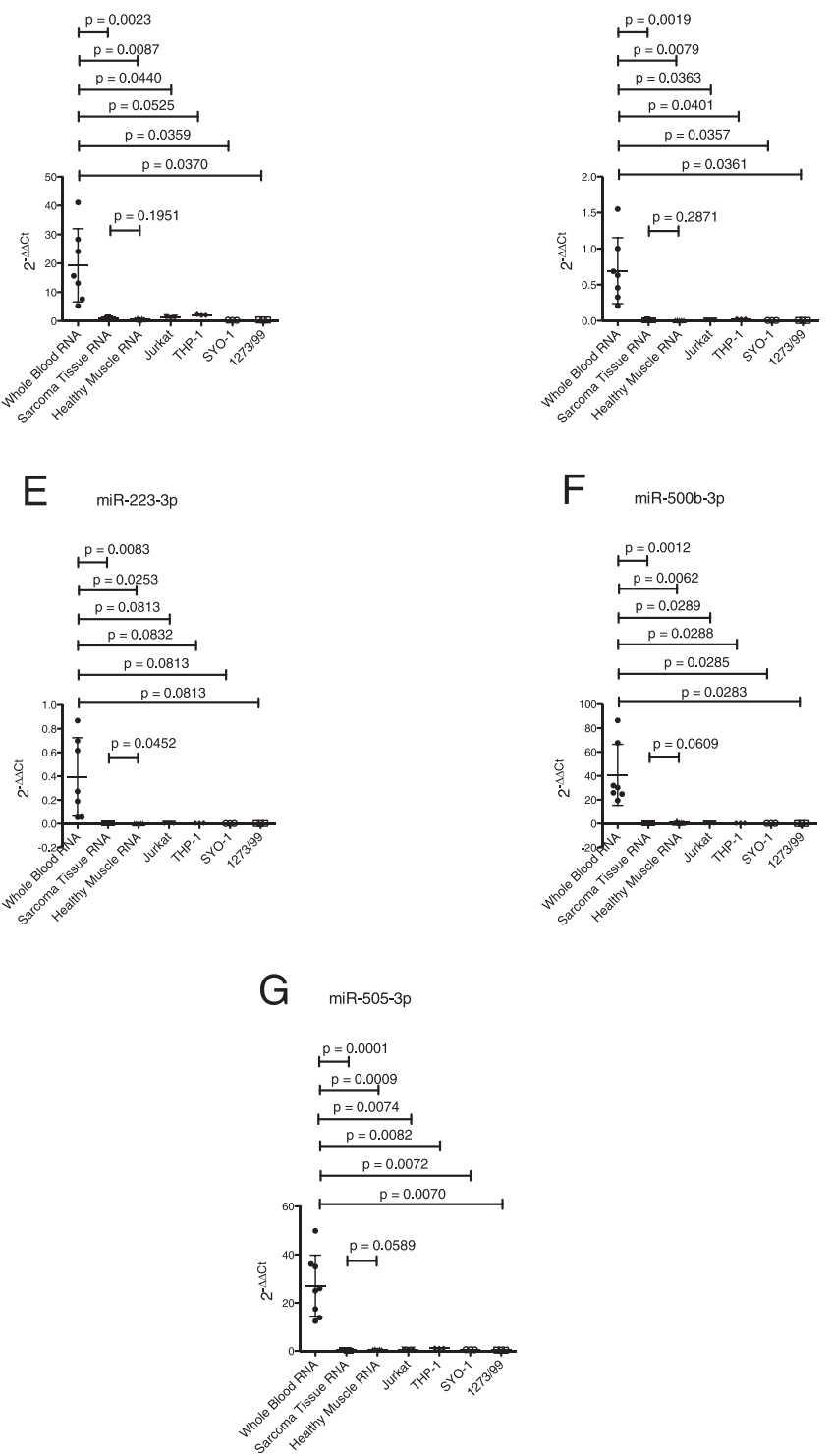

Fig. 5 Comparison of whole blood miRNA expression levels of patients with active synovial sarcoma to corresponding synovial sarcoma tissue, healthy skeletal muscle, Jurkat (human T-lymphocyte cell line), THP-1 cells (human acute monocytic leukemia cell line) and synovial sarcoma cells (SYO-1 und 1273/99 cell lines). a. miR-99a-5p. b. miR-146b-5p. c. miR-148b-3p. d. miR-195-5p. e. miR-223-3p. f. miR-500b-3p. g. miR-505-3p 
(Fig. 5a) and synovial sarcoma cells compared to THP-1 and Jurkat cells (Additional file 6A), thus indicating that the high expression levels of miR-99a-5p in synovial sarcoma patients might not result from a miRNA expression shift of peripheral blood cells but might actually be related to the tumor itself. However, miR-99a-5p shows overlapping expression values in whole blood of patients with active synovial sarcoma compared to the different control groups (Figs. 2 and 3), thus it is not an ideal biomarker for synovial sarcoma. Furthermore, it was shown that human macrophages transfer miR-223 to hepatocarcinoma cells through intercellular contact, leading to decreased expression of stathmin-1 and insulin-like growth factor-1 receptor and decreased proliferation of tumor cells [25], which adds to the point that the detected upregulation of miR-223-3p in whole blood cells might primarily be a defense of circulating mononuclear cells against the proliferation of synovial sarcoma.

Interestingly, we found that four of the detected seven miRNAs seem to interact with the nuclear factor kappa $\mathrm{B}$ pathway (NF- $\mathrm{kB}$ ), thus possibly playing an important role in toll like and cytokine receptor signaling. MiRNAs are well described regulators of the immune response [26]. The expression of miR-146b has been both shown to be upregulated by inflammation-related induction of NF- $\mathrm{kB}$ through a MyD88-dependent pathway, and also downregulating TNF receptor-associated factor 6 (TRAF6) and IL-1 receptor-associated kinase 1 (IRAK1), thus reducing the activity of the pathway by a negative feedback regulation loop and maintaining the balance of toll like and cytokine receptor signaling [27]. In consistency with this study, Hulsmans et al. showed that silencing of miR-146b-5p resulted in more NFkB p65 DNA binding activity and TNF $\alpha$. Moreover, low levels of miR-146b-5p in monocytes of obese persons have been shown to be associated with increased mitochondrial reactive oxygen species (ROS) [28], thus showing that miR-146b inhibits NF- $\mathrm{kB}$ - mediated inflammation. An upregulation of miR-146b-5p might thus reflect an immune evasion of the tumor, leading to increased tumor proliferation and progression. MiR195 also seems to interact with NF- $\mathrm{kB}$, as it was shown to decrease the expression of multiple NF- $\kappa B$ downstream effectors [29]. Another of the NFK-B related miRNAs is miR-223; as the transcriptional activity of miR-223 promoter was shown to be activated by Notch and NF-KB [30]. Finally, miR-500 was shown to induce gastric cancer cell proliferation and tumorigenicity by activating NF-kB. [31]. Thus, although finding the exact biological significance of the miRNAs in tumorigenesis of synovial sarcoma is a complex matter, it seems that the miRNAs detected in our study control various activities of the immune system by interacting with different pathways such as the NFK-B signaling pathway. This further underlines our hypothesis that the detected miRNA expression levels reflect changes of the immune system as a defense to tumor progression as well as immune evasion mechanisms of the tumor.

Biomarkers for synovial sarcoma are urgently needed to identify patients with local recurrence and metastatic disease, as imaging studies are often inconclusive. Although validated miRNAs were not always significantly different in patients with active disease compared to the different control groups, miR-146b-5p, miR-500b-3p and miR-505$3 p$ allow discrimination of patients of active synovial sarcoma patients from the different control groups, showing clear cut-off-values at approximately 18.5 (miR-146b-5p), 17.5 (miR-500b-3p) and 11.5 (miR505-3p) $2^{-\Delta \Delta C t}$; while miR-99-5p, miR-148b-3p, miR195-5p and miR-223-3p show overlapping expression values. These miRNAs are promising candidates as diagnostic biomarkers for synovial sarcoma, potentially allowing the detection of local recurrence or distant metastasis by a simple blood test: A rise of these miRNAs beyond the cut-off value - while initially lying underneath the cut-off value following complete removal of the tumor - might indicate local recurrence or distant metastasis, as shown in Fig. 4b (Patient 2). Moreover, these miRNAs could be applied in order to distinguish between synovial sarcoma and other types of sarcoma, which may be of therapeutic importance if chemosensitive sarcoma such as the rare extraosseous Ewing's sarcoma with spindle cell sarcoma-like pattern, often showing histological overlap with synovial sarcoma [32], are considered in differential diagnosis. Although our results proved to be highly significant, the main limitation of this study consists in the low number of patients within the different study groups. Nonetheless, one has to take into account that soft tissue sarcoma is a rare malignancy which is furthermore divided into a variety of different subtypes, thus making the collection of a large number of patients within one study group difficult. We preferred to identify a miRNA panel in a small group of patients with synovial sarcoma as an initial "proof of concept" rather than including a large number of patients with different sarcoma subtypes. As synovial sarcoma is characterized by the presence of the SS18-SSX fusion gene, which can be detected by fluorescence in situ hybridization (FISH) of tumor tissue, this sarcoma subtype can be specifically diagnosed without the risk of being confounded with other sarcoma subtypes. Through this approach, we achieved highly significant miRNA array and qRTPCR results and a clear separation of sarcoma samples from control samples by hierarchical clustering. The fact that the miRNA signature established in this pilot study even distinguishes synovial sarcoma from 
leiomyosarcoma, MPNST, Ewing sarcoma and liposarcoma samples further elucidates the specificity of our results.

Further studies are warranted to validate the panel of deregulated miRNAs in a larger cohort of independent synovial sarcoma patients and to identify miRNA signatures of other sarcoma subtypes. Moreover, the existing group of patients with synovial sarcoma in complete remission needs to be closely monitored in order to evaluate a possible rise of the established miRNAs with local recurrence or distant metastasis.

\section{Conclusion}

Our results have identified a specific whole blood miRNA signature that may serve as an independent biomarker for the diagnosis of local recurrence or distant metastasis of synovial sarcoma and that even distinguishes synovial sarcoma from other histological sarcoma subtypes.

\section{Methods}

\section{Study population}

All patients included in the study were patients receiving treatment from specialists in the interdisciplinary tumor board of the Comprehensive Cancer Center Freiburg (CCCF). Patients with a history of cancer other than sarcoma or any type of systemic inflammatory disease or autoimmune disorder were excluded. The control group included healthy adults matched to the active synovial sarcoma group in terms of age, sex and body mass index (BMI). Diagnosis of synovial sarcoma, leiomyosarcoma, MPNST, Ewing sarcoma and liposarcoma was confirmed by two independent pathologists.

\section{Ethics, consent and permissions}

Signed informed consent was obtained from all participants, allowing analysis of blood samples and all clinical data. The Ethics Committee of the Albert-LudwigsUniversity of Freiburg, Germany, approved the study. The design and performance of the study is in accordance with the Declaration of Helsinki.

\section{Blood sampling}

All blood samples were collected by puncture of the antecubital vein without tourniquet through a 20-gauge needle. The first $3 \mathrm{ml}$ of blood were discarded. Each $2.5 \mathrm{ml}$ of whole blood were collected and stabilized in PAXgene Blood RNA Tubes (PreAnalytiX, Hombrechtikon, Switzerland). The RNA Tubes were incubated for at least $2 \mathrm{~h}$ at room temperature after blood collection to ensure complete lysis of blood cells and were then stored at $-20{ }^{\circ} \mathrm{C}$ until further processing. Before starting the procedure, they were equilibrated to room temperature. Total RNA $>18$ nucleotides (including miRNA) was purified manually using the PAXgene Blood
miRNA Kit (PreAnalytiX) according to the manufacturer's protocol.

\section{miRNA Array}

RNA quality and quantity was assessed by capillary electrophoresis using the Fragment Analyzer and Standard/ High sensitivity RNA Analysis kits (Advanced Analytical Technologies, Ames, IA, U.S.). Total whole blood RNA of patients with active synovial sarcoma $(n=5)$ and healthy controls $(n=5)$ was then analyzed using an Affymetrix GeneChip miRNA Array v. 4.0 (Affymetrix, Santa Clara, CA, U.S.). $275 \mathrm{ng}$ of total RNA was labeled with Biotin using a 3DNA Array Detection FlashTag ${ }^{\text {Tn }}$ Biotin HSR kit (Genisphere, Hatfield, PA, U.S.) following the manufacturer's protocol, being subsequently hybridized overnight. The GeneChip miRNA 4.0 arrays, containing 30,424 total mature miRNA probe sets including 2.578 mature human miRNAs and miRNAs of 202 other organisms, were washed and stained using the Affymetrix GeneChip Hybridization Wash and Stain Kit and were then scanned with the Affymetrix GeneChip Scanner 3000 7G (Affymetrix, Santa Clara, CA, U.S.).

\section{Cell culture}

The samples of the human synovial sarcoma cell lines SYO-1 und 1273/99 were a donation from Dr. Marcus Renner, Institute of Pathology, University of Heidelberg.

SYO-1 synovial sarcoma cells were cultured in Dulbecco's modified minimal essential medium (life technologies, Carlsbad, CA, U.S.A.) supplemented with $10 \%$ FBS superior (Biochrom, Berlin, Germany), 100 U/ml PenicillinStreptomycin (life technologies) and 0.5 \% Sodium Pyruvate (Biochrom, Berlin, Germany).

1273/99 synovial sarcoma cells were cultured in F-12 Nutrient mixture (Ham) (life technologies) supplemented with $10 \%$ FBS superior and $100 \mathrm{U} / \mathrm{ml}$ PenicillinStreptomycin.

THP-1 cells (human acute monocytic leukemia cell line) and Jurkat cells (human T lymphocyte cell line) were cultured in RPMI Medium 1640 (with L-Glutamine; life technologies) supplemented with $10 \%$ FBS superior and 100 U/ml Penicillin-Streptomycin.

\section{RNA Isolation}

Total RNA of SYO-1, 1273/99, THP-1 and Jurkat cells was purified using the miRNeasy Mini Kit (Qiagen, Hilden, Germany) according to the manufacturer's protocol. Total RNA of tissue sample sections, each with a thickness of $10 \mu \mathrm{m}$ and a surface area of approximately $250 \mathrm{~mm}^{2}$, was purified after deparaffinization using the miRNeasy FFPE Kit (Qiagen) with an extension of the time of incubation with proteinase $\mathrm{K}$ at $56{ }^{\circ} \mathrm{C}$ from $15 \mathrm{~min}$ to $60 \mathrm{~min}$ for higher RNA yields. The remaining steps were carried out according to the manufacturer's protocol. The RNA was 
quantified using a Nanodrop 2000 (Thermo Fisher Scientific, Waltham, MA, USA).

\section{Preparation of cDNA}

For detection of miRNA expression levels, cDNA synthesis of whole blood RNA, cell RNA and tissue RNA (275 ng) was carried out using the miScript II RT Kit (Qiagen) according to the manufacturer's protocol. The reverse transcription reaction was incubated for $60 \mathrm{~min}$ at $37{ }^{\circ} \mathrm{C}$, followed by $5 \mathrm{~min}$ at $95{ }^{\circ} \mathrm{C}$ to inactivate the miScript Reverse Transcriptase Mix.

For detection of the SS18-SSX1 and SS18-SSX2 fusion gene transcript in tissue samples, each 275 ng of RNA was converted to cDNA using the AffinityScript Multi Temperature cDNA Synthesis Kit (Agilent Technologies, Santa Clara, CA, USA) according to the manufacturer's protocol. The reverse transcription reaction was incubated for $10 \mathrm{~min}$ at $25{ }^{\circ} \mathrm{C}$, followed by $1 \mathrm{~h}$ at $42{ }^{\circ} \mathrm{C}$ and 15 min at $70{ }^{\circ} \mathrm{C}$.

\section{qRT-PCR}

For detection of miRNA expression levels, qRT-PCR was performed using the miScript SYBR Green PCR Kit (Qiagen, Hilden, Germany) according to the manufacturer's protocol. Briefly, the cycling conditions consisted of an initial activation step of the HotStarTaq DNA Polymerase for $15 \mathrm{~min}$ at $95^{\circ} \mathrm{C}$, followed by 45 cycles of denaturation for $15 \mathrm{~s}$ at $94{ }^{\circ} \mathrm{C}$, annealing for $30 \mathrm{~s}$ at $55^{\circ} \mathrm{C}$ and extension for $30 \mathrm{~s}$ at $70{ }^{\circ} \mathrm{C}$ with fluorescence data collection being performed during the extension step. MiScript Primer Assays were purchased from Qiagen. $C t$-values were normalized to RNU6-2B, a frequently used reference gene [33] and $2^{-\Delta \Delta C t}$ values [34] were analyzed using Student's $t$-test for independent samples.

For detection of the SS18-SSX1 and SS18-SSX2 fusion gene transcript in tissue samples, qRT-PCR was performed using the Absolute qPCR ROX Mix (ThermoScientific, Waltham, MA, USA) and the following primers: SS18-SSX1 + FAM (Hs 03024820_ft), SS18SSX2 + FAM (Hs03024398_ft) (TaqMan Gene Expression Assays; Applied Biosystems by life technologies, Carlsbad, CA, USA) and the GAPDH-Primer Set (GAPDHprobe 899, GAPDH-875 F und GAPDH 946-R (Eurofins MWG Operon, Huntsville, AL, USA) as the internal control. Briefly, the cycling conditions were enzyme activation at $95{ }^{\circ} \mathrm{C}$ for $15 \mathrm{~min}$, followed by 50 cycles of denaturation at $95{ }^{\circ} \mathrm{C}$ for $15 \mathrm{~s}$ and annealing/extension at $60{ }^{\circ} \mathrm{C}$ for $1 \mathrm{~min}$. Ct-values $<36$ were considered as positive.

\section{Statistics}

For analysis of the miRNA array, CEL-files of the raw data were produced with Affymetrix GeneChip Command Console Software Version 4.0. (Affymetrix, Santa Clara,
CA, U.S.) Partek Genomics Suite software (Version 6.14.0923; Partek, Inc., St. Louis, MO, USA) was used for further analysis. CEL-files were imported including control and interrogating probes, and the arrays were normalized using quantile normalization. Probeset summarization was done using Median Polish. Probe values were $\log 2$ transformed. In order to detect differential miRNA expression between the 2 groups, 1-way ANOVA was performed [35] and Fisher's Least Significant Difference (LSD) was used as contrast method.

MultiExperiment Viewer 4.8 was used for hierarchical clustering of miRNA array data [36]. $2^{-\Delta \Delta C t}$ and patients' demographic data and blood counts were compared using Student's $t$-test for independent samples. p-values were rounded to 4 significant digits; $\mathrm{p}$-values below 0.05 were considered statistically significant.

\section{Additional files}

Additional file 1: Demographic patient data and blood count of patients with active sarcoma and control groups. Demographic patient data (age, BMI) and blood count (hemoglobin level, platelet count and leukocyte count) of patients with active synovial sarcoma compared to healthy donors, patients with synovial sarcoma in remission and patients with active leiomyosarcoma, MPNST, Ewing sarcoma and liposarcoma. Data are presented as mean value \pm standard error of mean (SEM). $p$ - values were determined using a Student's $t$-test for independent samples. $\mathrm{Hb}=$ Hemoglobin. BMl = Body Mass Index. (PPTX $68 \mathrm{~kb}$ )

Additional file 2: Demographic patient data and blood count of patients with active sarcoma (Individual Cohort (IC)) and control groups. Demographic patient data (age, BMI) and blood count (hemoglobin level, platelet count and leukocyte count) of patients with active synovial sarcoma (Individual Cohort (IC)) compared to healthy donors and patients with synovial sarcoma in remission. Data are presented as mean value \pm standard error of mean (SEM). $p$ - values were determined using a Student's $t$-test for independent samples. $\mathrm{Hb}=$ Hemoglobin. BMI = Body Mass Index. IC = Independent Cohort. (PPTX $66 \mathrm{~kb})$

Additional file 3: Disease and therapy status of sarcoma patients. $M_{0}=$ localized disease. $M_{1}=$ metastatic disease. Current chemotherapy/ radiotherapy involves treatment within the last 6 weeks. (PPTX 67 kb)

Additional file 4: Illustration of the miRNA-expression levels of the two active synovial sarcoma patients who received chemotherapy (Patient 2 and 3 of the screening cohort of active synovial sarcoma patients as depicted in Additional file 3) marked with a triangle, patients receiving anticoagulation (Patient 3 of the screening cohort of active synovial sarcoma patients and Patient 1 of the independent cohort of active synovial sarcoma patients as depicted in Additional file 3) marked in green and patients receiving radiotherapy/presenting localized disease (Patient 1 of the screening cohort of active synovial sarcoma patients as depicted in Additional file 3) marked in red. $A, C, E, G, I, K$ and $M$ include the screening cohort of active synovial sarcoma patients, $B, D, F, H, J, L$ and $N$ depict the independent cohort (IC) of synovial sarcoma patients. (JPEG $5044 \mathrm{~kb}$ )

Additional file 5: Comparison of whole blood miRNA expression levels of patients with active synovial sarcoma and miRNA expression levels of corresponding synovial sarcoma tissue. MiRNA-expression of patients with synovial sarcoma expressing SS18-SSX2 are marked in purple. A. miR-99a-5p. B. miR-146b-5p. C. miR-148b-3p. D. miR-195-5p. E. miR-223-3p. F. miR-500b-3p. G. miR-505-3p. (JPEG 2837 kb)

Additional file 6: MiRNA expression levels of Jurkat (human T-lymphocyte cell line) and THP-1 cells (human acute monocytic 
leukemia cell line) compared to synovial sarcoma cells (SYO-1 und 1273/99 cell lines). A. miR-99a-5p. B. miR-146b-5p. C. miR-148b-3p. D. miR-195-5p. E. miR-223-3p. F. miR-500b-3p. G. miR-505-3p. (JPEG 2926 kb)

\section{Abbreviations}

BMI: Body mass Index; CT: Computed Tomography; FISH: Fluorescence In Situ Hybridization; Hb: Hemoglobin; MiRNA: MicroRNA; MPNST: Malignant Peripheral Nerve Sheath Tumor; MRI: Magnetic Resonance Imaging; NF-kB: Nuclear factor k B; qRT-PCR: quantitative Reverse Transcription Polymerase Chain Reaction.

\section{Competing interests}

The authors declare that they have no competing interests.

\section{Authors' contributions}

AF, DB and SUE designed and guided the study. AF, PVU and DP performed and participated in analysis of laboratory experiments data. AF, PVU, JH, JS, GWH, $\mathrm{OH}$ and $\mathrm{PB}$ acquired and preserved clinical samples. AF drafted the manuscript. SUE, DB, HB and GBS provided administrative support and revised the manuscript. All authors have contributed and approved the final manuscript.

\section{Acknowledgements}

The authors thank Thomas Boschet, Dr. Sandra Strassburg and Prof. Dr. Günter Finkenzeller for their invaluable advice, Dr. Marcus Renner for the kind donation of synovial sarcoma cell lines SYO-1 and 1273/99 and Hannah Füllgraf for providing healthy skeletal muscle tissue samples. This study was supported by the Research Commission of the University of Freiburg, Faculty of Medicine (Grant Nr. 3095120017). Dr. Steffen U Eisenhardt is supported by a Heisenberg Fellowship of the German Research Foundation (DFG) (El866/3-1) and project grants El866/1-2 and El866/2-1 that are not related to the study.

\section{Author details \\ 'Department of Plastic and Hand Surgery, University Medical Center Freiburg, Hugstetter Strasse 55, 79106 Freiburg, Germany. ${ }^{2}$ Department of Hematology, Oncology and Stem Cell Transplantation, University Medical Center Freiburg, Hugstetter Strasse 55, 79106 Freiburg, Germany. ${ }^{3}$ Department of Radiation Oncology, University Medical Center Freiburg, Robert-Koch-Straße 3, 79106 Freiburg, Germany. ${ }^{4}$ Department of Orthopaedics and Traumatology, University Medical Center Freiburg, Hugstetter Strasse 55, 79106 Freiburg, Germany. ${ }^{5}$ Institute for Surgical Pathology, University Medical Center Freiburg, Breisacher Str. 115a, 79106 Freiburg, Germany. ${ }^{6}$ Tumorbank Comprehensive Cancer Center Freiburg, Breisacher Str. 115a, 79106 Freiburg, Germany.}

\section{Received: 16 March 2015 Accepted: 30 July 2015 Published online: 07 August 2015}

\section{References}

1. Zagars GK, Ballo MT, Pisters PW, Pollock RE, Patel SR, Benjamin RS, et al. Prognostic factors for patients with localized soft-tissue sarcoma treated with conservation surgery and radiation therapy: an analysis of 1225 patients. Cancer. 2003;97(10):2530-43.

2. Bannasch $H$, Eisenhardt SU, Grosu AL, Heinz J, Momeni A, Stark GB. The diagnosis and treatment of soft tissue sarcomas of the limbs. Dtsch ArztebI Int. 2011;108(3):32-8

3. Eilber FC, Dry SM. Diagnosis and management of synovial sarcoma. J Surg Oncol. 2008;97(4):314-20.

4. Crew AJ, Clark J, Fisher C, Gill S, Grimer R, Chand A, et al. Fusion of SYT to two genes, SSX1 and SSX2, encoding proteins with homology to the Kruppel-associated box in human synovial sarcoma. EMBO J. 1995; 14(10):2333-40.

5. Clark J, Rocques PJ, Crew AJ, Gill S, Shipley J, Chan AM, et al. Identification of novel genes, SYT and SSX, involved in the $t(X ; 18)$ (p11.2; q11.2) translocation found in human synovial sarcoma. Nat Genet. 1994:7(4):502-8.

6. Kawai A, Woodruff J, Healey JH, Brennan MF, Antonescu CR, Ladanyi M. SYT-SSX gene fusion as a determinant of morphology and prognosis in synovial sarcoma. N Engl J Med. 1998;338(3):153-60.
7. Skytting B, Nilsson G, Brodin B, Xie Y, Lundeberg J, Uhlen M, et al. A novel fusion gene, SYT-SSX4, in synovial sarcoma. J Natl Cancer Inst. 1999;91(11):974-5

8. Amary MF, Berisha F, Bernardi Fdel C, Herbert A, James M, Reis-Filho JS, et al. Detection of SS18-SSX fusion transcripts in formalin-fixed paraffinembedded neoplasms: analysis of conventional RT-PCR, GRT-PCR and dual color FISH as diagnostic tools for synovial sarcoma. Mod Pathol. 2007;20(4):482-96.

9. Doench JG, Sharp PA. Specificity of microRNA target selection in translational repression. Genes Dev. 2004;18(5):504-11.

10. Nonaka R, Nishimura J, Kagawa Y, Osawa H, Hasegawa J, Murata K, et al. Circulating miR-199a-3p as a novel serum biomarker for colorectal cancer. Oncol Rep. 2014;32(6):2354-8.

11. Chen X, Ba Y, Ma L, Cai X, Yin Y, Wang K, et al. Characterization of microRNAs in serum: a novel class of biomarkers for diagnosis of cancer and other diseases. Cell Res. 2008;18(10):997-1006.

12. Juan $L$, Tong HL, Zhang P, Guo G, Wang Z, Wen X, et al. Identification and characterization of novel serum microRNA candidates from deep sequencing in cervical cancer patients. Sci Rep. 2014:4:6277.

13. Lu J, Getz G, Miska EA, Alvarez-Saavedra E, Lamb J, Peck D, et al. MicroRNA expression profiles classify human cancers. Nature. 2005;435(7043):834-8.

14. Ugras S, Brill E, Jacobsen A, Hafner M, Socci ND, Decarolis PL, et al. Small RNA sequencing and functional characterization reveals MicroRNA-143 tumor suppressor activity in liposarcoma. Cancer Res. 2011;71(17):5659-69.

15. Subramanian S, Lui WO, Lee CH, Espinosa I, Nielsen TO, Heinrich MC et al. MicroRNA expression signature of human sarcomas. Oncogene. 2008;27(14):2015-26

16. Renner M, Czwan E, Hartmann W, Penzel R, Brors B, Eils R, et al. MicroRNA profiling of primary high-grade soft tissue sarcomas. Genes Chromosomes Cancer. 2012;51(11):982-96.

17. Miyachi M, Tsuchiya K, Yoshida H, Yagyu S, Kikuchi K, Misawa A, et al. Circulating muscle-specific microRNA, miR-206, as a potential diagnostic marker for rhabdomyosarcoma. Biochem Biophys Res Commun. 2010:400(1):89-93.

18. Hisaoka M, Matsuyama A, Nagao Y, Luan L, Kuroda T, Akiyama H, et al. Identification of altered MicroRNA expression patterns in synovial sarcoma. Genes Chromosomes Cancer. 2011;50(3):137-45.

19. Storey J. A direct approach to false discovery rates. J R Stat Soc Series B Stat Methodol. 2002;64(3):479-98.

20. Cheng HH, Yi HS, Kim Y, Kroh EM, Chien JW, Eaton KD, et al. Plasma processing conditions substantially influence circulating microRNA biomarker levels. PLoS One. 2013;8(6), e64795.

21. Keller A, Leidinger P, Bauer A, Elsharawy A, Haas J, Backes C, et al. Toward the blood-borne miRNome of human diseases. Nat Methods. 2011:8(10):841-3.

22. Mookherjee N, El-Gabalawy HS. High degree of correlation between whole blood and PBMC expression levels of miR-155 and miR-146a in healthy controls and rheumatoid arthritis patients. J Immunol Methods. 2013;400-401:106-10.

23. Leidinger P, Backes C, Dahmke IN, Galata V, Huwer H, Stehle I, et al. What makes a blood cell based miRNA expression pattern disease specific?-a miRNome analysis of blood cell subsets in lung cancer patients and healthy controls. Oncotarget. 2014;5(19):9484-97.

24. Fujiwara T, Kunisada T, Takeda K, Uotani K, Yoshida A, Ochiya T, et al. MicroRNAs in soft tissue sarcomas: overview of the accumulating evidence and importance as novel biomarkers. Biomed Res Int 2014;2014:592868

25. Aucher A, Rudnicka D, Davis DM. MicroRNAs transfer from human macrophages to hepato-carcinoma cells and inhibit proliferation. J Immunol. 2013:191(12):6250-60.

26. Eisenhardt SU, Weiss JB, Smolka C, Maxeiner J, Pankratz F, Bemtgen X, et al. MicroRNA-155 aggravates ischemia-reperfusion injury by modulation of inflammatory cell recruitment and the respiratory oxidative burst. Basic Res Cardiol. 2015;110(3):32.

27. Taganov KD, Boldin MP, Chang KJ, Baltimore D. NF-kappaB-dependent induction of microRNA miR-146, an inhibitor targeted to signaling proteins of innate immune responses. Proc Natl Acad Sci U S A. 2006;103(33):12481-6.

28. Hulsmans M, Van Dooren E, Mathieu C, Holvoet P. Decrease of miR-146b-5p in monocytes during obesity is associated with loss of the anti-inflammatory but not insulin signaling action of adiponectin. PLoS One. 2012;7(2), e32794. 
29. Ding J, Huang S, Wang Y, Tian Q, Zha R, Shi H, et al. Genome-wide screening reveals that miR-195 targets the TNF-alpha/NF-kappaB pathway by down-regulating IkappaB kinase alpha and TAB3 in hepatocellular carcinoma. Hepatology. 2013;58(2):654-66.

30. Kumar V, Palermo R, Talora C, Campese AF, Checquolo S, Bellavia D, et al. Notch and NF-kB signaling pathways regulate miR-223/FBXW7 axis in T-cell acute lymphoblastic leukemia. Leukemia. 2014;28(12):2324-35.

31. Zhang L, Ding Y, Yuan Z, Liu J, Sun J, Lei F, et al. MicroRNA-500 sustains nuclear factor-kappaB activation and induces gastric cancer cell proliferation and resistance to apoptosis. Oncotarget. 2015;6(4):2483-95.

32. Pinto A, Dickman P, Parham D. Pathobiologic markers of the ewing sarcoma family of tumors: state of the art and prediction of behaviour. Sarcoma. 2011;2011:856190.

33. Ma $Y$, Wang $X$, Jin H. Methylated DNA and microRNA in Body Fluids as Biomarkers for Cancer Detection. Int J Mol Sci. 2013;14(5):10307-31.

34. Livak KJ, Schmittgen TD. Analysis of relative gene expression data using real-time quantitative PCR and the 2(-Delta Delta C(T)) Method. Methods. 2001;25(4):402-8.

35. Eisenhart $\mathrm{C}$. The assumptions underlying the analysis of variance. Biometrics. 1947;3(1):1-21.

36. Saeed Al, Sharov V, White J, Li J, Liang W, Bhagabati N, et al. TM4: a free, open-source system for microarray data management and analysis. Biotechniques. 2003;34(2):374-8

\section{Submit your next manuscript to BioMed Central and take full advantage of:}

- Convenient online submission

- Thorough peer review

- No space constraints or color figure charges

- Immediate publication on acceptance

- Inclusion in PubMed, CAS, Scopus and Google Scholar

- Research which is freely available for redistribution 